\section{Diferenciais nos fatores de risco para a mortalidade infantil em cinco cidades brasileiras: um estudo de caso-controle com base no SIM e no SINASC}

\author{
Differences in risk factors for infant mortality in five \\ Brazilian cities: a case-control study based on the \\ Mortality Information System and Information \\ System on Live Births
}

\author{
1 Centro de Pesquisas Aggeu \\ Magalhães, Fundação \\ Oswaldo Cruz, Recife, Brasil. \\ Correspondência \\ L. T. S. Maia \\ Centro de Pesquisas Aggeu \\ Magalhães, Fundação \\ Oswaldo Cruz. \\ Av. Professor Moraes Rego s/n, \\ Campus da UFPE - Cidade \\ Universitária, Recife, $P E$ \\ 50670-420, Brasil. \\ livia_tsouza@yahoo.com.br
}

\begin{abstract}
This study aimed to identify differences in risk factors for infant mortality in five cities, one from each region of Brazil. This was a case-control study with cases defined as deaths in infants less than one year of age in the Mortality Information System (SIM) and Information System on Live Births (SINASC) and controls as live born infants recorded in the SINASC database and who had not died in the first year. Risk factors were estimated by univariate and multivariate analysis, using hierarchical logistic models. The main determinants of infant mortality were biological factors (low Apgar score, low birth weight, prematurity, and congenital malformations) mediated by socioeconomic factors (education, marital status, and race/color) and prenatal care conditions (prenatal visits). Despite agreement on the determinants of infant mortality among the five cities, some regional differences were observed, expressing infant mortality associated with inequalities in socioeconomic conditions and access to health services.
\end{abstract}

Infant Mortality; Health Inequalities; Information Systems; Risk Factors
Lívia Teixeira de Souza Maia 1

Wayner Vieira de Souza 1

Antonio da Cruz Gouveia Mendes 1

\section{Introdução}

O óbito infantil como ocorrência "evitável” por serviços de saúde eficazes constitui-se em um "evento sentinela" da qualidade da atenção à saúde. Em países onde o risco de morrer dos menores de um ano permanece elevado, a necessidade de se obter indicadores de qualidade que evidenciem esta problemática não é apenas uma exigência metodológica, mas ética, por que implica a "mortalidade consentida" de crianças. Assim, a desigualdade do risco de ocorrência dos problemas de saúde é também uma medida da evitabilidade dos eventos e, consequentemente, da qualidade dos sistemas de saúde 1 .

O monitoramento da mortalidade infantil e de seus fatores de risco é essencial para identificar possíveis impactos de mudanças sociais e econômicas e dos avanços, e eventuais retrocessos, da cobertura e da qualidade dos serviços de saúde. O estudo dos fatores de risco dos óbitos em menores de um ano, compreendidos como indicadores de várias dimensões das condições de vida, possibilita elucidar elementos da cadeia de eventos determinantes, identificar grupos expostos a diferentes fatores e detectar diferenciadas necessidades de saúde em subgrupos populacionais, subsidiando as intervenções voltadas à redução dos óbitos infantis 1 .

A mortalidade infantil e seus determinantes têm sido objeto de muitos estudos no Brasil, constatando-se nos últimos anos um aumento 
considerável de publicações. Uma revisão sistemática da literatura científica sobre mortalidade infantil, em três importantes bases de dados [MEDLINE (Medical Literature Analysis and Retrieval System Online), LILACS (Literatura LatinoAmericana em Ciências de Saúde) e PAHO (Pan American Health Organization)], abrangendo o período de 1998 a 2006, analisou 59 publicações relevantes sobre o tema 2 .

Esta importante produção científica deve-se, pelo menos em parte, a um progressivo aperfeiçoamento dos sistemas de informações em saúde do país, especificamente o Sistema de Informações sobre Mortalidade (SIM) e o Sistema de Informações sobre Nascidos Vivos (SINASC), resultando numa maior cobertura e qualidade das informações 3 .

Aliados ao desenvolvimento das principais bases de dados, alguns estudos têm realizado a integração dos diversos sistemas de informação em saúde, principalmente quanto à inclusão de novos indicadores para a gerência destes sistemas, na perspectiva do aumento da notificação e da qualidade da informação 4,5,6,7.

Embora seja possível constatar importantes avanços no estudo da mortalidade infantil, especialmente em virtude da melhoria da qualidade das informações, e apesar de não se pretender esgotar a discussão desse tema complexo, a identificação da existência de desigualdades regionais na distribuição dos óbitos infantis e nos fatores de risco permite revelar as dificuldades e as potencialidades dos sistemas de informações, auxiliando a vigilância epidemiológica e subsidiando uma melhor integração entre os sistemas de informação e serviços que a compõem.

Nesse sentido o presente estudo teve como objetivo identificar diferenciais entre os fatores de risco associados à mortalidade infantil em cinco cidades brasileiras, sendo uma de cada macrorregião, com base nas informações oriundas do SIM e do SINASC.

\section{Metodologia}

O critério inicial para escolha das cidades foi a semelhança de porte populacional. Em seguida realizou-se uma avaliação da qualidade dos dados do SIM e do SINASC, a partir de indicadores de cobertura e qualidade dos dados, utilizando as informações do Sistema de Monitoramento dos Indicadores de Mortalidade Infantil (MonitorIMI/Fiocruz. http://www.monitorimi.cict.fio cruz.br/fontes.htm, acessado em 10/Nov/2008). Sendo, portanto, escolhidas as seguintes cidades: Belém (Pará), Recife (Pernambuco), Guarulhos (São Paulo), Porto Alegre (Rio Grande do Sul) e
Goiânia (Goiás). Todas as cidades selecionadas apresentam $100 \%$ de cobertura de nascidos vivos e óbitos infantis, possibilitando o cálculo do coeficiente de mortalidade infantil estimado através do método direto.

Foi realizado um estudo caso-controle para cada cidade, no qual foram considerados como casos os óbitos de menores de um ano ocorridos entre 1 o de janeiro a 31 de dezembro de 2005 registrados no SIM e como controles os nascidos vivos que não foram a óbito entre 1o de janeiro de 2004 e 31 de dezembro de 2005 registrados no SINASC.

Foram selecionados dois anos para os dados do SINASC, para que houvesse a possibilidade de resgatar todas as Declarações de Nascidos Vivos referentes aos óbitos infantis do ano de 2005 , sendo necessária a inclusão dos nascimentos no ano anterior ao óbito (2004).

A seleção dos casos partiu dos óbitos registrados no SIM e pareados com o SINASC, sendo incluídos no estudo apenas os óbitos pareados com a Declaração de Nascido Vivo correspondente. Os óbitos não pareados foram excluídos do estudo.

Os controles foram obtidos por meio de amostra aleatória simples dos nascidos vivos que não foram a óbito, e, portanto, tendo como critério de inclusão não ter sido pareado com o banco do SIM.

O tamanho da amostra foi calculado para permitir detectar, em cada cidade, significância de odds ratio (OR) maior que 2,0, considerando um poder do estudo (1-ß) de $80 \%$, um erro alfa de $5 \%$ e uma frequência relativa de $10 \%$ de exposição entre os controles, adotando-se como fator o baixo peso ao nascer. Optou-se pela seleção de três controles para um caso em cada cidade. Por tanto seria necessário em cada cidade cerca de 210 casos e 630 controles.

Para garantir que os controles fossem selecionados da mesma população dos casos, adotou-se como critério, além do município de residência, o estabelecimento de saúde onde ocorreu o nascimento da criança. Os estabelecimentos foram categorizados em estratos de acordo com o número de nascidos vivos. Neste caso, a partir dos estratos definidos, foram identificados os números de casos e foi retirada uma amostra de três controles para cada caso de todos os estabelecimentos categorizados pelo critério, independente da ocorrência do óbito. Para definição dos estratos, foi realizada uma análise descritiva da distribuição do número de nascidos vivos por estabelecimento de saúde de cada uma das cidades estudadas.

As variáveis independentes foram categorizadas para realização da análise hierarquizada considerando os modelos teóricos de determinação 
da mortalidade infantil, em três níveis 8: (1) distal (variáveis socioeconômicas) - escolaridade da mãe, estado civil da mãe, ocupação materna e raça/cor; (2) intermediário (variáveis relacionadas à atenção à saúde materno-infantil) - local do nascimento, tipo de parto, número de consultas de pré-natal e idade da mãe; (3) proximal (variáveis biológicas da mãe e do recém-nascido) - ín-

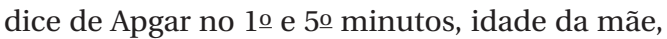
tipo da gravidez, sexo da criança, peso ao nascer, duração da gestação, paridade materna (número de filhos nascidos vivos e mortos e presença de malformação congênita).

Realizou-se também uma análise da completitude de cada uma das variáveis do estudo, sendo incluídas apenas as variáveis com completitude maior ou igual a $80 \%$.

Para verificar a existência de desigualdades regionais em termos de mortalidade infantil, construiu-se uma medida de risco (risco relativo - RR) dividindo-se taxas observadas em cada cidade pela taxa média de mortalidade do país (valor esperado) 9 .

As duas bases de dados (SIM e SINASC) foram relacionadas através do linkage em duas etapas (linkage determinístico e probabilístico) 5 .

O linkage determinístico foi realizado por meio da variável unívoca constante nas duas bases de dados (SIM e SINASC), o número da Declaração de Nascido Vivo (NUMERODN), uma vez que, para os óbitos de menores de um ano, essa variável deve estar preenchida na Declaração de Óbito.

Na segunda etapa foi realizado linkage probabilístico entre os registros de óbitos de menores de um ano com os registros de nascimento que não foram pareados na primeira etapa do linkage (determinístico), tendo sido utilizado o programa Reclink III (versão 3.0.4.4005 para a unificação das bases de dados 5 .

A análise dos dados se deu em duas etapas: análise univariada e análise multivariada hierarquizada (regressão logística).

$\mathrm{Na}$ análise univariada verificou-se a associação entre o desfecho e cada variável independente, calculando-se as OR brutas e respectivos intervalos de 95\% de confiança (IC95\%), com respectivo cálculo da significância estatística do qui-quadrado de Pearson.

Em seguida, realizou-se a análise multivariada, incluindo nesta etapa as variáveis independentes cujas associações foram significantes a um nível menor ou igual a $20 \%$ na análise univariada e adotando-se como critério a estratégia proposta por Victora et al. 8, que utiliza modelos hierarquizados.

A hierarquização das exposições na regressão logística foi feita de acordo com um modelo teórico que contempla as inter-relações entre os diversos grupos de fatores. De acordo com o modelo utilizado, fatores nos níveis superiores da hierarquia influenciam o desfecho através de um efeito independente e/ou um efeito intermediado por fatores nos níveis inferiores. Isto se traduz na prática de análise, pela avaliação do efeito dos fatores de interesse ajustados apenas para os fatores de confusão pertencentes a um nível hierárquico igual ou superior ao seu.

Portanto, a regressão logística foi realizada em cinco etapas: as três primeiras etapas referem-se às regressões multivariadas dentro de cada bloco (distal, intermediário e proximal). Em cada uma das etapas foram incluídas as variáveis que na análise univariada apresentassem $\mathrm{p}<0,20$; permanecendo no modelo multivariado final de cada bloco as variáveis que mantivessem $\mathrm{p}<0,10$. $\mathrm{Na}$ quarta etapa foram incorporadas as variáveis do modelo final do nível distal àquelas do modelo final do nível intermediário. Foram retiradas do modelo final dessa etapa tão somente as variáveis do nível intermediário que passaram a apresentar $\mathrm{p}>0,10$. Na quinta e última etapa juntou-se às variáveis do modelo final anterior (quarta etapa) as variáveis do nível proximal. Sendo também nessa última etapa retiradas apenas as variáveis do nível proximal que apresentaram $p>0,10$, considerando os pressupostos da análise hierarquizada.

A regressão logística foi realizada por meio do programa SPSS 13.0 for Windows (SPSS Inc., Chicago, Estados Unidos). Foram obtidas as OR ajustadas com seus respectivos IC95\%, que compuseram o modelo final de cada cidade.

\section{Resultados}

Entre as cinco cidades estudadas, no ano de 2005, Porto Alegre foi a que apresentou a menor taxa de mortalidade infantil (12,9/1.000 nascidos vivos), seguida pelas cidades de Goiânia e Guarulhos, 13,7 e 14,7/1.000 nascidos, respectivamente. Enquanto que Belém foi a cidade com maior coeficiente de mortalidade infantil dentre as analisadas (20,0/1.000 nascidos vivos) (Tabela 1$)$.

O cálculo do RR para o coeficiente de mortalidade infantil nas cinco cidades estudadas demonstra a existência de um gradiente de desigualdades regionais na ocorrência dos óbitos em menores de um ano.

Tendo como padrão o Brasil $(\mathrm{RR}=1,00)$, observa-se que a cidade de Belém apresenta RR mais elevado $(1,18)$. Enquanto que as cidades de Guarulhos, Porto Alegre e Goiânia apresentaram o risco relativo menor que 1 , indicando taxas menores do que a apresentada no país. Apenas para 
Risco relativo (RR) por cidade do Brasil para o coeficiente de mortalidade infantil (CMI) no ano de 2005.

\begin{tabular}{lcccccc}
\hline Local & Óbitos & $\begin{array}{c}\text { Nascidos } \\
\text { vivos }\end{array}$ & CMI & RR & IC95\% & Valor de $p$ \\
\hline Brasil (referência) & 51.544 & 3.035 .096 & 17,0 & 1,00 & - & - \\
Belém & 472 & 23.544 & 20,0 & 1,18 & $1,08-1,29$ & $<0,001$ \\
Recife & 385 & 23.221 & 16,6 & 0,98 & $0,88-1,08$ & 0,660 \\
Guarulhos & 316 & 21.489 & 14,7 & 0,87 & $0,78-0,97$ & 0,012 \\
Porto Alegre & 244 & 18.944 & 12,9 & 0,76 & $0,67-0,86$ & $<0,001$ \\
Goiânia & 268 & 19.631 & 13,7 & 0,81 & $0,72-0,91$ & $<0,001$ \\
\hline
\end{tabular}

IC95\%: intervalo de $95 \%$ de confiança.

a cidade de Recife esta diferença não foi estatisticamente significante (Tabela 1).

Dos 1.685 óbitos de menores de um ano nas cinco cidades estudadas, após a realização do linkage dos bancos de dados do SIM e do SINASC, foi possível relacionar 1.516 (90\%) Declarações de Óbito à sua respectiva Declaração de Nascido Vivo. Apenas 169 (10\%) não foram pareadas.

Porto Alegre foi a cidade onde se obteve o maior percentual de óbitos pareados com a respectiva Declaração de Nascido Vivo (96,7\%). Em Guarulhos e em Recife foi possível relacionar $96,2 \%$ e $95,6 \%$ dos óbitos de menores de um ano, respectivamente. Nas cidades de Belém e Goiânia foram obtidos os menores percentuais de óbitos pareados, $81,6 \%$ e $83,2 \%$, respectivamente.

Em relação ao tipo de linkage realizado, do total dos óbitos pareados nas cinco cidades (1.516), $1.130(74,5 \%)$ foram relacionados por meio do linkage determinístico, evidenciando a qualidade das informações das referidas bases de dados, destacando-se as cidades de Porto Alegre e Recife, com uma proporção de até 99,6\% e 97\% de registros pareados por meio do linkage determinístico, respectivamente. Guarulhos foi, entre as cidades estudadas, a que apresentou menor percentual de pares obtidos por meio do linkage determinístico, apenas 16,8\%, 51 óbitos do total de 304 registros pareados. Porém, foi também em Guarulhos onde houve a maior contribuição do linkage probabilístico, 83,2\% correspondendo a 253 registros do total de 304 pares.

Como produto final das etapas do linkage obteve-se o grupo de casos, constituído por 1.516 óbitos pareados com a respectiva Declaração de Nascido Vivo e o grupo de controles selecionados por meio de uma amostra 4.548 crianças de um universo de 213.241 Declarações de Nascidos Vivos não pareadas com a Declaração de Óbito, assim distribuídos: 385 casos e 1.155 controles em Belém; 368 casos e 1.104 controles em Recife; 304 casos e 912 controles em Guarulhos; 236 casos e 708 controles em Porto Alegre; 223 casos e 669 controles em Goiânia. Valores esses superiores aos mínimos estipulados para o tamanho da amostra.

A Tabela 2 apresenta a distribuição de frequência das variáveis independentes para casos e controles nas cinco cidades.

A Tabela 3 apresenta o resultado da análise univariada da associação entre as variáveis de exposição e o desfecho das cinco cidades, sendo consideradas significativas aquelas com $\mathrm{p}<$ 0,20

No nível distal destaca-se que a escolaridade da mãe não foi significativamente associada com o desfecho em Guarulhos e Goiânia, que a ocupação da mãe só apresentou significância em Recife e que o estado civil da mãe só não foi significantemente associado com o desfecho em Belém.

Dentre as variáveis do nível intermediário, o número de consultas no pré-natal esteve significativamente associado ao desfecho em todas as cinco cidades, apresentando OR bruta superior a cinco em três cidades e superior a oito nas outras duas cidades. O parto vaginal se constituiu como fator de risco nas cidades de Belém, Recife e Goiânia e apenas em Porto Alegre foi encontrada associação com o local de ocorrência do nascimento e a mortalidade infantil. A idade da mãe se constituiu como fator de risco em todas as cidades com exceção do Recife.

Para o nível proximal, observou-se associação significativa com o desfecho para as variáveis Apgar 1o minuto, Apagar 5o minuto, tipo de gravidez, peso ao nascer, duração da gestação e malformação congênita, todas com OR bastante elevadas. 
Tabela 2

Número de casos e controles das cinco cidades brasileiras estudadas em 2005.

\begin{tabular}{|c|c|c|c|c|c|c|c|c|c|c|}
\hline \multirow[t]{2}{*}{ Variáveis } & \multicolumn{2}{|c|}{ Belém } & \multicolumn{2}{|c|}{ Recife } & \multicolumn{2}{|c|}{ Guarulhos } & \multicolumn{2}{|c|}{ Porto Alegre } & \multicolumn{2}{|c|}{ Goiânia } \\
\hline & Casos & Controles & Casos & Controles & Casos & Controles & Casos & Controles & Casos & Controles \\
\hline \multicolumn{11}{|l|}{ Nível distal } \\
\hline \multicolumn{11}{|l|}{ Escolaridade da mãe (anos } \\
\hline \multicolumn{11}{|l|}{ de estudo) } \\
\hline$<4$ & 40 & 69 & 38 & 76 & 19 & 46 & 19 & 27 & 8 & 15 \\
\hline $4-7$ & 132 & 391 & 149 & 388 & 105 & 293 & 86 & 223 & 56 & 195 \\
\hline $8 e+$ & 212 & 695 & 173 & 620 & 172 & 565 & 130 & 446 & 132 & 407 \\
\hline \multicolumn{11}{|l|}{ Ocupação da mãe } \\
\hline Sem ocupação & 309 & 917 & 291 & 809 & 215 & 635 & 112 & 354 & 100 & 314 \\
\hline Com ocupação & 76 & 237 & 75 & 285 & 85 & 267 & 79 & 267 & 63 & 178 \\
\hline \multicolumn{11}{|l|}{ Estado civil da mãe } \\
\hline Casada & 64 & 218 & 73 & 347 & 95 & 343 & 46 & 180 & 82 & 303 \\
\hline Solteira/Separada/Viúva & 320 & 937 & 290 & 702 & 194 & 532 & 180 & 493 & 126 & 330 \\
\hline \multicolumn{11}{|l|}{ Raça/Cor } \\
\hline Branca & 67 & 214 & 67 & 396 & 44 & 134 & 168 & 575 & 100 & 372 \\
\hline Parda/Preta & 318 & 940 & 292 & 700 & 18 & 42 & 68 & 131 & 32 & 119 \\
\hline \multicolumn{11}{|l|}{ Nível intermediário } \\
\hline \multicolumn{11}{|l|}{ Local ocorrência do } \\
\hline \multicolumn{11}{|l|}{ nascimento } \\
\hline Domicílio & 1 & 0 & 1 & 2 & 2 & 0 & 8 & 9 & & \\
\hline Hospital & 384 & 1.155 & 367 & 1.102 & 302 & 912 & 227 & 695 & 223 & 669 \\
\hline \multicolumn{11}{|l|}{ Tipo de parto } \\
\hline Cesário & 166 & 593 & 142 & 538 & 154 & 453 & 114 & 311 & 109 & 379 \\
\hline Vaginal & 219 & 562 & 226 & 566 & 148 & 459 & 122 & 397 & 113 & 288 \\
\hline \multicolumn{11}{|l|}{ Número de consultas } \\
\hline $0-3$ & 113 & 97 & 137 & 115 & 76 & 68 & 85 & 86 & 35 & 18 \\
\hline $4-6$ & 146 & 435 & 141 & 407 & 85 & 241 & 63 & 160 & 54 & 121 \\
\hline $7 e+$ & 123 & 594 & 80 & 574 & 122 & 586 & 88 & 461 & 120 & 497 \\
\hline \multicolumn{11}{|l|}{ Idade da mãe (anos) } \\
\hline $10-19$ & 108 & 282 & 92 & 92 & 70 & 151 & 55 & 117 & 59 & 117 \\
\hline $20-34$ & 246 & 807 & 250 & 788 & 193 & 651 & 131 & 487 & 141 & 495 \\
\hline $35 e+$ & 31 & 66 & 26 & 87 & 41 & 110 & 50 & 104 & 20 & 54 \\
\hline \multicolumn{11}{|l|}{ Nível proximal } \\
\hline \multicolumn{11}{|l|}{ Apgar 10 minuto } \\
\hline $0-3$ & 107 & 22 & 138 & 13 & 71 & 24 & 81 & 16 & 78 & 7 \\
\hline $4-7$ & 106 & 128 & 111 & 139 & 118 & 143 & 82 & 97 & 74 & 91 \\
\hline $8-10$ & 172 & 1.004 & 119 & 944 & 104 & 729 & 73 & 585 & 69 & 564 \\
\hline Apgar 5 o minuto & & & & & & & & & & \\
\hline $0-3$ & 37 & 4 & 80 & 3 & 29 & 2 & 28 & 1 & 28 & 3 \\
\hline $4-7$ & 91 & 29 & 93 & 22 & 88 & 22 & 72 & 23 & 77 & 13 \\
\hline $8-10$ & 257 & 1.121 & 195 & 1.074 & 179 & 880 & 136 & 674 & 117 & 650 \\
\hline Tipo de gravidez & & & & & & & & & & \\
\hline Múltipla & 22 & 22 & 29 & 19 & 31 & 20 & 22 & 5 & 19 & 10 \\
\hline Única & 363 & 1.133 & 339 & 1.085 & 272 & 890 & 214 & 703 & 204 & 658 \\
\hline Sexo & & & & & & & & & & \\
\hline Feminino & 171 & 583 & 160 & 521 & 137 & 437 & 117 & 325 & 95 & 338 \\
\hline Masculino & 211 & 572 & 207 & 583 & 166 & 475 & 119 & 381 & 127 & 329 \\
\hline
\end{tabular}

(continua) 


\begin{tabular}{|c|c|c|c|c|c|c|c|c|c|c|}
\hline \multirow[t]{2}{*}{ Variáveis } & \multicolumn{2}{|c|}{ Belém } & \multicolumn{2}{|c|}{ Recife } & \multicolumn{2}{|c|}{ Guarulhos } & \multicolumn{2}{|c|}{ Porto Alegre } & \multicolumn{2}{|c|}{ Goiânia } \\
\hline & Casos & Controles & Casos & Controles & Casos & Controles & Casos & Controles & Casos & Controles \\
\hline \multicolumn{11}{|l|}{ Nível proximal } \\
\hline \multicolumn{11}{|c|}{ Peso ao nascer (gramas) } \\
\hline$<2.500$ & 236 & 122 & 232 & 87 & 196 & 91 & 152 & 75 & 132 & 50 \\
\hline $2.500 \mathrm{e}+$ & 149 & 1.033 & 136 & 1.017 & 106 & 816 & 84 & 633 & 85 & 618 \\
\hline \multicolumn{11}{|c|}{ Duração da gestação } \\
\hline \multicolumn{11}{|l|}{ (semanas) } \\
\hline$<37$ & 203 & 61 & 229 & 95 & 175 & 73 & 140 & 76 & 106 & 32 \\
\hline $37 e+$ & 182 & 1.094 & 138 & 1.009 & 114 & 788 & 96 & 631 & 88 & 621 \\
\hline \multicolumn{11}{|c|}{ Paridade (filhos nascidos } \\
\hline \multicolumn{11}{|l|}{ vivos) } \\
\hline 0 & 169 & 123 & 176 & 511 & 119 & 361 & 99 & 327 & 126 & 287 \\
\hline 1 & 99 & 385 & 93 & 336 & 82 & 285 & 61 & 201 & 67 & 219 \\
\hline $2 e+$ & 96 & 243 & 99 & 233 & 90 & 234 & 75 & 179 & 30 & 124 \\
\hline \multicolumn{11}{|c|}{ Paridade (filhos nascidos } \\
\hline \multicolumn{11}{|l|}{ mortos) } \\
\hline 0 & 264 & 295 & 318 & 978 & 238 & 762 & 215 & 673 & 192 & 546 \\
\hline 1 & 60 & 132 & 32 & 67 & 30 & 71 & 14 & 31 & 19 & 57 \\
\hline $2 e+$ & 17 & 27 & 16 & 20 & 12 & 17 & 7 & 3 & 10 & 20 \\
\hline \multicolumn{11}{|c|}{ Malformação congênita } \\
\hline Sim & 40 & 3 & 42 & 8 & 39 & 8 & 15 & 55 & 21 & 3 \\
\hline Não & 345 & 1.151 & 326 & 1.096 & 163 & 637 & 691 & 180 & 167 & 575 \\
\hline
\end{tabular}

\section{Tabela 3}

Valores da odds ratio bruta (OR) e da significância (valor de p) obtidos mediante análise univariada para associação entre os óbitos de menores de um ano e os fatores de risco para as cinco cidades brasileiras estudadas em 2005

\begin{tabular}{|c|c|c|c|c|c|c|c|c|c|c|}
\hline \multirow[t]{2}{*}{ Variáveis } & \multicolumn{2}{|c|}{ Belém } & \multicolumn{2}{|c|}{ Recife } & \multicolumn{2}{|c|}{ Guarulhos } & \multicolumn{2}{|c|}{ Porto Alegre } & \multicolumn{2}{|c|}{ Goiânia } \\
\hline & OR & Valor de $p$ & OR & Valor de $\mathrm{p}$ & OR & Valor de $p$ & OR & Valor de $p$ & OR & Valor de $p$ \\
\hline \multicolumn{11}{|l|}{ Nível distal } \\
\hline $\begin{array}{l}\text { Escolaridade da mãe } \\
\text { (anos de estudo) }\end{array}$ & & 0,011 & & 0,005 & & 0,358 & & 0,013 & & 0,390 \\
\hline$<4$ & 1,90 & & 1,79 & & 1,36 & & 2,41 & & 1,64 & \\
\hline $4-7$ & 1,10 & & 1,38 & & 1,18 & & 1,26 & & 0,88 & \\
\hline $8 \mathrm{e}+$ & 1,00 & & 1,00 & & 1,00 & & 1,00 & & 1,00 & \\
\hline Ocupação da mãe & & 0,737 & & 0,033 & & 0,676 & & 0,69 & & 0,571 \\
\hline Sem ocupação & 1,05 & & 1,37 & & 1,06 & & 1,06 & & 0,90 & \\
\hline Com ocupação & 1,00 & & 1,00 & & 1,00 & & 1,00 & & 1,00 & \\
\hline Estado civil da mãe & & 0,333 & & $<0,001$ & & 0,054 & & 0,055 & & 0,034 \\
\hline Casada & 1,00 & & 1,00 & & 1,00 & & 1,00 & & 1,00 & \\
\hline $\begin{array}{l}\text { Solteira/Separada/ } \\
\text { Viúva }\end{array}$ & 1,16 & & 1,96 & & 1,31 & & 1,42 & & 1,41 & \\
\hline Raça/Cor & & 0,616 & & $<0,001$ & & 0,420 * & & $<0,001$ & & 0,999 * \\
\hline Branca & 1,00 & & 1,00 & & 1,00 & & 1,00 & & 1,00 & \\
\hline Parda/Preta & 1,08 & & 2,46 & & 1,30 & & 1,77 & & 1,01 & \\
\hline
\end{tabular}

(continua) 
Tabela 3 (continuação)

\begin{tabular}{|c|c|c|c|c|c|c|c|c|c|c|}
\hline \multirow[t]{2}{*}{ Variáveis } & \multicolumn{2}{|c|}{ Belém } & \multicolumn{2}{|c|}{ Recife } & \multicolumn{2}{|c|}{ Guarulhos } & \multicolumn{2}{|c|}{ Porto Alegre } & \multicolumn{2}{|c|}{ Goiânia } \\
\hline & OR & Valor de $\mathrm{p}$ & OR & Valor de $p$ & OR & Valor de $p$ & OR & Valor de $p$ & OR & Valor de $p$ \\
\hline \multicolumn{11}{|l|}{ Nível intermediário } \\
\hline Local ocorrência do & & & & 0,739 & & & & 0,034 & & \\
\hline \multicolumn{11}{|l|}{ nascimento } \\
\hline Domicílio & & & 1,50 & & & & 2,72 & & & \\
\hline Hospital & & & 1,00 & & & & 1,00 & & & \\
\hline Tipo de parto & & 0,005 & & $<0,001$ & & 0,69 & & 0,242 & & 0,045 \\
\hline Cesário & 1,00 & & 1,00 & & 1,00 & & 1,00 & & 1,00 & \\
\hline Vaginal & 1,39 & & 1,51 & & 0,95 & & 0,84 & & 1,36 & \\
\hline Número de consultas & & $<0,001$ & & $<0,001$ & & $<0,001$ & & $<0,001$ & & $<0,001$ \\
\hline $0-3$ & 5,63 & & 8,55 & & 5,37 & & 5,18 & & 8,05 & \\
\hline $4-6$ & 1,52 & & 2,49 & & 1,69 & & 2,06 & & 1,85 & \\
\hline $7 e+$ & 1,00 & & 1,00 & & 1,00 & & & & 1,00 & \\
\hline Idade da mãe (anos) & & 0,064 & & 0,224 & & 0,022 & & $<0,001$ & & 0,008 \\
\hline $10-19$ & 1,26 & & 0,94 & & 1,56 & & 1,75 & & 1,77 & \\
\hline $20-34$ & 1,00 & & 1,00 & & 1,00 & & 1,00 & & 1,00 & \\
\hline $35 e+$ & 1,54 & & 1,27 & & 1,26 & & 1,79 & & 1,30 & \\
\hline \multicolumn{11}{|l|}{ Nível proximal } \\
\hline Apgar 1ㅇ minuto & & $<0,001$ & & $<0,001$ & & $<0,001$ & & $<0,001$ & & $<0,001$ \\
\hline $0-3$ & 28,39 & & 84,21 & & 20,74 & & 40,57 & & 91,08 & \\
\hline $4-7$ & 4,83 & & 6,33 & & 5,78 & & 6,77 & & 6,65 & \\
\hline $8-10$ & 1,00 & & 1,00 & & 1,00 & & 1,00 & & 1,00 & \\
\hline Apgar 5 o minuto & & $<0,001$ & & $<0,001$ & & $<0,001$ & & $<0,001$ & & $<0,001$ \\
\hline $0-3$ & 40,35 & & 146,87 & & 71,28 & & 138,76 & & 51,85 & \\
\hline $4-7$ & 13,69 & & 23,28 & & 19,66 & & 15,51 & & 32,91 & \\
\hline $8-10$ & 1,00 & & 1,00 & & 1,00 & & 1,00 & & 1,00 & \\
\hline Tipo de gravidez & & $<0,001$ & & $<0,001$ & & $<0,001$ & & $<0,001$ & & $<0,001$ \\
\hline Múltipla & 3,12 & & 4,88 & & 5,07 & & 14,45 & & 6,13 & \\
\hline Única & 1,00 & & 1,00 & & 1,00 & & 1,00 & & 1,00 & \\
\hline Sexo & & 0,053 & & 0,231 & & 0,414 & & 0,345 & & 0,042 \\
\hline Feminino & 1,00 & & 1,00 & & 1,00 & & 1,00 & & 1,00 & \\
\hline Masculino & 1,26 & & 1,16 & & 1,11 & & 0,87 & & 1,37 & \\
\hline $\begin{array}{l}\text { Peso ao nascer } \\
\text { (gramas) }\end{array}$ & & $<0,001$ & & $<0,001$ & & $<0,001$ & & $<0,001$ & & $<0,001$ \\
\hline$<2.500$ & 13,41 & & 19,94 & & 16,58 & & 15,27 & & 19,19 & \\
\hline $2.500 \mathrm{e}+$ & 1,00 & & 1,00 & & 1,00 & & 1,00 & & 1,00 & \\
\hline $\begin{array}{l}\text { Duração da gestação } \\
\text { (semanas) }\end{array}$ & & $<0,001$ & & $<0,001$ & & $<0,001$ & & $<0,001$ & & $<0,001$ \\
\hline$<37$ & 20,00 & & 17,62 & & 16,57 & & 12,11 & & 23,37 & \\
\hline $37 e+$ & 1,00 & & 1,00 & & 1,00 & & 1,00 & & 1,00 & \\
\hline Paridade (filhos & & $<0,001$ * & & 0,38 & & 0,257 & & 0,144 & & 0,013 \\
\hline \multicolumn{11}{|l|}{ nascidos vivos) } \\
\hline 0 & 5,34 & & 1,24 & & 1,14 & & 0,99 & & 1,43 & \\
\hline 1 & 1,00 & & 1,00 & & 1,00 & & 1,00 & & 1,00 & \\
\hline $2 e+$ & 1,54 & & 1,53 & & 1,34 & & 1,38 & & 0,79 & \\
\hline Paridade (filhos & & $<0,001$ * & & 0,009 & & 0,052 & & 0,01 & & 0,653 \\
\hline \multicolumn{11}{|l|}{ nascidos mortos) } \\
\hline 0 & 1,00 & & 1,00 & & 1,35 & & 1,00 & & 1,00 & \\
\hline 1 & 0,70 & & 1,47 & & 2,26 & & 1,41 & & 0,95 & \\
\hline $2 e+$ & 0,51 & & 2,24 & & 1,00 & & 7,30 & & 1,42 & \\
\hline Malformação & & $<0,001 *$ & & $<0,001$ & & $<0,001$ * & & $<0,001$ & & $<0,001$ \\
\hline \multicolumn{11}{|l|}{ congênita } \\
\hline Sim & 44,48 & & 17,65 & & 19,05 & & 14,08 & & 24,10 & \\
\hline Não & 1,00 & & 1,00 & & 1,00 & & 1,00 & & 1,00 & \\
\hline
\end{tabular}

* Variáveis não incluídas por apresentarem completitude inferior a 80\%. 
A gestação múltipla representou risco nas cinco cidades, o sexo masculino em Belém e Goiânia e ser criança parda e preta em Recife e Porto Alegre. A paridade de dois ou mais filhos vivos em Porto Alegre e mães sem filhos nascidos vivos em Goiânia e a paridade de filhos nascidos mortos em Recife, Porto Alegre e Guarulhos apresentaram maior valor da OR.

Após a análise univariada as variáveis que apresentaram valor de p acima de $20 \%$ ou completitude inferior a $80 \%$ foram retiradas do modelo. Foram excluídas nessa etapa, por apresentarem completitude inferior a $80 \%$, as variáveis raça/cor nas cidades de Guarulhos e Goiânia, número de filhos nascidos vivos e mortos em Belém e a variável presença de malformações congênitas em Guarulhos.

As variáveis assim selecionadas foram introduzidas no modelo multivariado por blocos, obedecendo à ordem hierárquica e as etapas descritas na metodologia.

As etapas intermediárias da análise hierarquizada (etapas um a quatro) e respectivos valores de significância (valor de p) para cada variável das cinco cidades estudadas são apresentadas na Tabela 4. Ressalta-se que a última etapa não é apresentada nessa tabela, uma vez que representa o modelo final apresentado na Tabela 5. Esta tabela resume os achados da análise da regressão logística multivariada para associação entre

Tabela 4

Etapas intermediárias da análise hierarquizada e respectivos valores de significância (valor de p) para cada variável das cinco cidades brasileiras estudadas.

\begin{tabular}{|c|c|c|c|c|c|}
\hline \multirow[t]{2}{*}{ Variáveis/Etapas da análise multivariada } & \multicolumn{5}{|c|}{ Valor de $p$} \\
\hline & Belém & Recife & Guarulhos & Porto Alegre & Goiânia \\
\hline \multicolumn{6}{|l|}{ Etapa 1 (distal) } \\
\hline Escolaridade da mãe & 0,011 & 0,364 & & 0,046 & \\
\hline Ocupação da mãe & - & 0,691 & & & \\
\hline Estado civil da mãe & - & 0,005 & 0,055 & 0,157 & 0,034 \\
\hline Raça/Cor & - & 0,000 & & 0,020 & \\
\hline \multicolumn{6}{|l|}{ Etapa 2 (intermediário) } \\
\hline Local ocorrência do nascimento & - & & & 0,892 & \\
\hline Tipo de parto & 0,784 & 0,917 & & & 0,819 \\
\hline Número de consultas & 0,000 & 0,000 & 0,000 & 0,000 & 0,000 \\
\hline Idade da mãe & 0,030 & & 0,092 & 0,002 & 0,130 \\
\hline \multicolumn{6}{|l|}{ Etapa 3 (proximal) } \\
\hline Apgar 1ㅇ minuto & 0,001 & 0,000 & 0,004 & 0,001 & 0,000 \\
\hline Apgar 5o minuto & 0,000 & 0,003 & 0,000 & 0,000 & 0,003 \\
\hline Tipo de gravidez & 0,121 & 0,331 & 0,861 & 0,003 & 0,969 \\
\hline Sexo & 0,124 & & & & 0,775 \\
\hline Peso ao nascer & 0,000 & 0,000 & 0,000 & 0,000 & 0,012 \\
\hline Duração da gestação & 0,000 & 0,003 & 0,000 & 0,124 & 0,000 \\
\hline Filhos nascidos vivos & - & & & 0,101 & 0,843 \\
\hline Filhos nascidos mortos & - & 0,126 & 0,195 & 0,134 & \\
\hline Malformação congênita & 0,000 & 0,000 & & 0,000 & 0,001 \\
\hline \multicolumn{6}{|l|}{ Etapa 4 (distal e intermediário) } \\
\hline Escolaridade da mãe & 0,192 * & & & $0,221 *$ & \\
\hline Ocupação da mãe & - & & & & \\
\hline Estado civil da mãe & - & 0,132 & 0,738 * & & 0,391 * \\
\hline Raça/Cor & - & 0,000 & & 0,166 * & \\
\hline Local ocorrência do nascimento & - & & & & \\
\hline Tipo de parto & - & & & & \\
\hline Número de consultas & 0,000 & 0,000 & 0,073 & 0,000 & 0,000 \\
\hline Idade da mãe & 0,032 & & 0,000 & 0,004 & \\
\hline
\end{tabular}

Nota: [-]: excluídas na análise univariada; $[p<0,10]$ : mantidas no modelo; $[p>0,10]$ : retiradas do modelo; [*]: mantidas no modelo apesar de perderem a significância. 
Tabela 5

Valores de odds ratio ajustada $\left(\mathrm{OR}_{\text {ajustada }}\right)$ e significância (valor de p) obtidos mediante análise de regressão logística para associação entre os óbitos de menores de um ano e os fatores de risco para as cinco cidades brasileiras estudadas em 2005.

\begin{tabular}{|c|c|c|c|c|c|c|c|c|c|c|}
\hline \multirow[t]{2}{*}{ Variáveis } & \multicolumn{2}{|c|}{ Belém } & \multicolumn{2}{|c|}{ Recife } & \multicolumn{2}{|c|}{ Guarulhos } & \multicolumn{2}{|c|}{ Porto Alegre } & \multicolumn{2}{|c|}{ Goiânia } \\
\hline & $\mathrm{OR}_{\text {ajustada }}$ & $\begin{array}{l}\text { Valor } \\
\text { de } p\end{array}$ & $\mathrm{OR}_{\text {ajustada }}$ & $\begin{array}{l}\text { Valor } \\
\text { de } p\end{array}$ & $\mathrm{OR}_{\text {ajustada }}$ & $\begin{array}{l}\text { Valor } \\
\text { de p }\end{array}$ & $\mathrm{OR}_{\text {ajustada }}$ & $\begin{array}{l}\text { Valor } \\
\text { de } p\end{array}$ & $\mathrm{OR}_{\text {ajustada }}$ & $\begin{array}{l}\text { Valor } \\
\text { de } p\end{array}$ \\
\hline \multicolumn{11}{|l|}{ Nível distal } \\
\hline $\begin{array}{l}\text { Escolaridade da mãe (anos de } \\
\text { estudo) }\end{array}$ & & 0,001 & & & & & & 0,309 & & 0,061 \\
\hline$<4$ & 2,72 & & & & & & 2,11 & & 21,90 & \\
\hline $4-7$ & 1,44 & & & & & & 1,28 & & 2,99 & \\
\hline $8 e+$ & 1,00 & & & & & & 1,00 & & 1,00 & \\
\hline Estado civil da mãe & & & & 0,005 & & 0,296 & & & & \\
\hline Casada & & & 1,00 & & 1,00 & & & & & \\
\hline Solteira/Separada/Viúva & & & 1,83 & & 1,25 & & & & & \\
\hline Raça/Cor & & & & 0,016 & & & & 0,343 & & \\
\hline Branca & & & 1,00 & & & & 1,00 & & & \\
\hline Parda/Preta & & & 1,65 & & & & 1,28 & & & \\
\hline \multicolumn{11}{|l|}{ Nível intermediário } \\
\hline Número de consultas & & 0,036 & & 0,007 & & 0,184 & & 0,004 & & 0,392 \\
\hline $0-3$ & 1,85 & & 2,04 & & 1,29 & & 2,56 & & 1,88 & \\
\hline $4-6$ & 1,10 & & 1,04 & & 0,75 & & 1,12 & & 0,76 & \\
\hline $7 \mathrm{e}+$ & 1,00 & & 1,00 & & 1,00 & & 1,00 & & 1,00 & \\
\hline Idade da mãe (anos) & & 0,061 & & & & 0,395 & & 0,228 & & \\
\hline $10-19$ & 1,95 & & & & 1,30 & & 1,61 & & & \\
\hline $20-34$ & 1,00 & & & & 1,00 & & 1,00 & & & \\
\hline $35 e+$ & 0,92 & & & & 1,33 & & 1,12 & & & \\
\hline \multicolumn{11}{|l|}{ Nível proximal } \\
\hline Apgar 1으 minuto & & 0,001 & & 0,000 & & 0,003 & & 0,001 & & 0,000 \\
\hline $0-3$ & 3,62 & & 7,49 & & 2,56 & & 3,74 & & 21,90 & \\
\hline $4-7$ & 1,72 & & 3,01 & & 2,05 & & 2,59 & & 2,99 & \\
\hline $8-10$ & 1,00 & & 1,00 & & 1,00 & & 1,00 & & 1,00 & \\
\hline Apgar 5 o minuto & & 0,001 & & 0,021 & & 0,000 & & 0,002 & & 0,032 \\
\hline $0-3$ & 5,33 & & 6,48 & & 20,70 & & 39,20 & & 6,89 & \\
\hline $4-7$ & 3,15 & & 2,29 & & 3,74 & & 3,09 & & 3,33 & \\
\hline $8-10$ & 1,00 & & 1,00 & & 1,00 & & 1,00 & & 1,00 & \\
\hline \multicolumn{11}{|l|}{ Tipo de gravidez } \\
\hline \multicolumn{11}{|l|}{ Múltipla } \\
\hline \multicolumn{11}{|l|}{ Única } \\
\hline Peso ao nascer (gramas) & & 0,000 & & 0,000 & & 0,000 & & 0,000 & & 0,036 \\
\hline$<2.500$ & 3,17 & & 4,54 & & 3,43 & & 6,17 & & 2,49 & \\
\hline $2.500 \mathrm{e}+$ & 1,00 & & 1,00 & & 1,00 & & 1,00 & & 1,00 & \\
\hline Duração da gestação (semanas) & & 0,000 & & 0,005 & & 0,000 & & & & 0,000 \\
\hline$<37$ & 5,29 & & 2,20 & & 3,78 & & & & 5,53 & \\
\hline $37 e+$ & 1,00 & & 1,00 & & 1,00 & & & & 1,00 & \\
\hline Malformação congênita & & 0,000 & & 0,002 & & & & 0,000 & & 0,002 \\
\hline Sim & 26,19 & & 4,69 & & & & 24,40 & & 10,89 & \\
\hline Não & 1,00 & & 1,00 & & & & 1,00 & & 1,00 & \\
\hline
\end{tabular}


os óbitos de menores de um ano e os fatores de risco nas cinco cidades estudadas, apresentando os valores da OR ajustada e da significância estatística, permitindo verificar os diferenciais existentes.

Dentre as variáveis do nível distal permaneceram no modelo multivariado a escolaridade materna em Belém e Porto Alegre; o estado civil da mãe em Recife, Guarulhos e Goiânia e raça/ cor em Recife e Porto Alegre. Saliente-se que a escolaridade da mãe e raça/cor em Porto Alegre e o estado civil da mãe em Guarulhos permaneceram no modelo final apesar de apresentarem valores de $\mathrm{p}>0,10$ pelas razões conceituais já explicitadas.

Do nível intermediário o número de consultas de pré-natal constituiu o modelo final em todas as cidades. A idade materna foi significante nas cidades de Belém, Guarulhos e Porto Alegre.

No que se refere ao nível proximal, compuseram o modelo de risco as variáveis: índice Apgar no 10 e 5 o minutos e peso ao nascer nas cinco cidades estudadas, tipo de gravidez apenas na cidade de Porto Alegre. A duração da gestação foi significativa em todas as cidades, exceto em Porto Alegre. A presença de malformação apresentou significância estatística em todas as cidades, exceto em Guarulhos onde essa variável não foi testada por apresentar completitude inferior a $80 \%$.

\section{Discussão}

Os achados do presente estudo revelam marcantes desigualdades no perfil da mortalidade infantil. A maior mortalidade em Belém e a menor em Porto Alegre evidencia que tais diferenças estão fortemente associadas às condições socioeconômicas e de acesso aos serviços de saúde, constituindo-se em um indicador de iniquidade.

Dentre os fatores distais (socioeconômicos), o nível de escolaridade da mãe apresentou associação com a mortalidade infantil apenas nas cidades de Belém e Porto Alegre, verificando-se uma maior probabilidade de mortes em menores de um ano entre as crianças nascidas de mães com baixa instrução. Esse dado assume relevância uma vez que a escolaridade materna é tida como um marcador da condição socioeconômica da mãe e de sua família, relacionando-se nesse contexto com o perfil cultural e comportamental, ligados aos cuidados de saúde, agindo como importante determinante das mortes infantis 10,11 .

Uma investigação dos fatores associados à mortalidade infantil evitável na capital gaúcha no triênio de 2000 a 2003 destaca que entre o gru- po de casos houve um maior número de mães com até três anos de escolaridade 12 . Resultados semelhantes foram encontrados numa coorte de nascidos vivos em Maracanaú (Ceará), ao se constatar uma tendência de diminuição do risco à medida que o grau de escolaridade materna (em anos de estudo) aumentava 13

Ser mãe solteira, viúva ou separada judicialmente, representaram fatores de risco para a mortalidade infantil nas cidades de Recife, Guarulhos e Goiânia. Corrobora esses achados o estudo realizado em Porto Alegre no triênio de 2000 a 2003 demonstrando que as crianças nascidas de mães casadas tiveram maiores chances de sobrevivência do que as de famílias com mães em união consensual ou sozinhas ${ }^{14}$. A ausência de companheiro pode indicar uma maior vulnerabilidade social, uma lacuna no suporte emocional e econômico para a família ${ }^{12}$. Esta variável permanece no modelo final para Guarulhos, porém perdendo significância provavelmente pela mais forte associação apresentada pelas variáveis dos níveis intermediário e proximal.

A variável raça/cor da criança representou fator de risco para a mortalidade infantil nas cidades de Recife e Porto Alegre. Nessas cidades as crianças pretas e pardas apresentaram maior risco de morte no primeiro ano de vida. Ressaltase que nas cidades de Guarulhos e Goiânia essa associação não pôde ser testada em virtude da baixa completitude da variável.

A determinação da mortalidade infantil pela raça/cor, enquanto variável relacionada às condições socioeconômicas, encontrada no presente estudo corrobora os achados da literatura que apontam as diferenças étnicas entre brancos e negros como preditoras de risco, caracterizando uma condição iníqua de saúde e demonstrando a necessidade da adoção de políticas públicas de inclusão e equidade que busquem estreitar o fosso racial $15,16,17$.

A atenção à saúde da mãe e da criança tem sido apontada como fator de grande relevância para a redução da mortalidade infantil. As variáveis reprodutivas maternas e as relacionadas às condições de nascimento da criança permitem a avaliação da qualidade dessa atenção 12,18.

A frequência às consultas de pré-natal tem sido demonstrada por alguns estudos como uma das variáveis mais importantes relacionadas à gestação e ao parto na prevenção da morbidade e mortalidade infantil e de seus componentes 1,13,19,20. O acompanhamento rigoroso durante o pré-natal permite a identificação e intervenção precoces no sentido de minimizar danos à saúde materno-infantil. Sendo assim, a garantia de assistência pré-natal de qualidade e organizada de forma a garantir acessibilidade à gestante, pode 
detectar doenças maternas e fetais, melhorando assim a possibilidade de sobrevivência do recémnascido e reduzindo a prevalência de retardo do crescimento intrauterino, a prematuridade e a ocorrência de baixo peso ao nascer 1,19,20.

Nesse estudo, o baixo número de consultas de pré-natal esteve associado à mortalidade infantil em todas as análises realizadas, configurando-se como importante fator de risco para a mortalidade infantil. Essas constatações corroboram a literatura científica 13,21,22,23,24. Ressalvem-se apenas os casos de Guarulhos e Goiânia onde esta variável permanece no modelo final pela hierarquização adotada, com valores de p ligeiramente superiores a $10 \%$, provavelmente pela colinearidade com variáveis do nível proximal mais fortemente associadas com o desfecho.

A variável idade da mãe demonstrou-se associada ao desfecho em três das cidades analisadas. A existência de maior probabilidade de morte infantil entre os nascidos vivos de mães com idades consideradas extremas, ou seja, com idade inferior a 20 anos e naquelas de 35 anos e mais é sugerida em outros estudos 4,12,24. Destaca-se ainda a maior razão de exposição entre os nascidos vivos de mães adolescentes. Esses achados demonstram a necessidade de fortalecer as ações de planejamento familiar preconizadas pela atenção primária à saúde.

O índice de Apgar no 1o e no 5o minutos é utilizado na mensuração da vitalidade do nascido vivo, constituindo-se em um importante indicador de risco para a morbimortalidade perinatal 23 . Os achados do estudo demonstram que os mais baixos escores desse índice, tanto no lo como no 5o minuto, constituíram-se como fator de risco no modelo final de determinação da mortalidade infantil nas cinco cidades estudadas, corroborando vários estudos 13,22,23,25,26,27,28.

Quanto ao tipo de gravidez, na análise univariada foi possível observar entre as mães com gravidez múltipla uma maior razão de exposição entre o grupo de casos do que entre os controles para todas as cidades estudadas. Porém na interação com outras variáveis ajustadas no modelo multivariado por meio da regressão logística, o tipo de gravidez perde a significância estatística em quase todas as cidades, permanecendo no modelo multivariado final apenas da cidade de Porto Alegre, corroborando os resultados de outros estudos 12,22.

A associação entre gravidez múltipla e mortalidade neonatal pode ter sido esvaziada na análise multivariada, provavelmente em virtude do fato de a idade gestacional e o baixo peso ao nascer, muito prevalente entre os gemelares, serem variáveis independentes mais fortemente associadas à mortalidade 22 .
O baixo peso ao nascer demonstrou forte associação com as mortes em menores de um ano em todas as análises realizadas, permanecendo no modelo final de determinação da mortalidade infantil das cinco cidades estudas.

A associação entre o baixo peso ao nascer e a mortalidade de menores de um ano, semelhantemente ao encontrado neste estudo, é bastante divulgada na literatura científica. Os estudos relatam que tanto para a mortalidade neonatal como pós-neonatal, o baixo peso ao nascer é o fator individual mais fortemente associado ao óbito infantil 10,11,12,13,21,22,24,26,29,30.

No que se refere à duração da gestação os resultados do estudo apontam para uma forte associação entre prematuridade (duração da gestação inferior a 37 semanas) e a mortalidade infantil. Essa variável esteve presente no modelo final de risco em quatro cidades estudadas, à exceção apenas de Porto Alegre.

Muitos dos fatores que concorrem para os partos prematuros são originados na mudança do estilo de vida nas últimas décadas, tais como o uso de álcool e fumo, estresse social e ocupacional, nutrição inadequada e outras condições em que as intervenções para uma gestação saudável deveriam ocorrer bem antes da concepção 31 .

A prematuridade tem como consequência uma situação de anormalidade no parto, exigindo um maior investimento em recursos tecnológicos e humanos adequados para o atendimento em tais circunstâncias. A falta de unidades de terapias intensivas pode dificultar a prevenção de óbitos potencialmente evitáveis entre os prematuros 22 .

A presença de malformações congênitas configurou-se como fator de risco fortemente associado à mortalidade infantil em todas as cidades à exceção de Guarulhos, onde essa variável só não foi analisada por possuir completitude inferior a $80 \%$. Em outro estudo realizado na capital gaúcha a presença de malformação congênita esteve mais fortemente associada à mortalidade não-evitável 12 .

Apesar da maior determinação da mortalidade infantil pelos fatores biológicos, destacase a importância da condição socioeconômica e da assistência pública à saúde da gestante e do recém-nascido como determinantes para a mortalidade infantil, uma vez que são fatores passíveis de mudança garantidas por políticas públicas de qualidade e um crescimento social da população.

Os fatores biológicos, principalmente o índice de Apgar no 1o e 5o minutos, baixo peso ao nascer, a prematuridade e a presença de malformação congênita, são situações não mais reversíveis no momento do parto. A determinação so- 
cioeconômica representada pela escolaridade da mãe e a condição de ser mãe solteira, separada ou viúva, raça/cor assim como, a constatação de que a grande maioria das gestantes realiza um número insuficiente de consultas pré-natais e o número de mães com idades extremas demonstraram estar associados com o desfecho. Além de esses achados representarem graves constatações, também se relacionam a condições reversíveis com a melhoria das condições sociais da população e um maior investimento e qualificação da assistência às mulheres e crianças.

Deve-se neste ponto destacar que a utilização de uma abordagem hierarquizada para análise dessas associações demonstrou-se como forma conceitualmente coerente de detectar esta estrutura de determinação do desfecho, mantendo no modelo final as variáveis explicativas baseando-se não apenas no critério da significância estatística. E embora não tenha sido factível a construção de um modelo teórico mais rebuscado em função da disponibilidade dos dados para as unidades analisadas, o desafio posto foi construir o melhor modelo possível a partir dos dados disponíveis.

Outro aspecto que merece destaque é que a seleção das cinco cidades, sendo uma de cada macrorregião brasileira, foi uma alternativa para apreender as diferenças regionais. Embora não representando toda a complexidade dessa questão, os achados desse trabalho apontam para importantes diferenciais regionais entre os fatores de risco para a mortalidade infantil.

Por fim, ressalta-se que a utilização da técnica de linkage foi de grande valia possibilitando resgatar na declaração de nascido vivo as variáveis preditoras da mortalidade infantil e, viabilizando a realização do desenho de estudo analítico tipo caso-controle. Além disso, ressalte-se a importante contribuição da utilização dos dados oriundos nos sistemas de informação em saúde disponíveis no Brasil, reduzindo os custos na realização, além de demonstrar o potencial de aplicação dessa estratégia na vigilância do óbito infantil nos serviços de saúde.

\section{Resumo}

O estudo buscou identificar diferenciais entre os fatores de risco para mortalidade infantil em cinco cidades, sendo uma de cada macrorregião brasileira. Realizou-se um estudo caso-controle, considerando casos os óbitos de menores de um ano registrados no Sistema de Informações sobre Mortalidade (SIM) e no Sistema de Informações sobre Nascidos Vivos (SINASC) e controles os nascidos vivos que não foram a óbito, registrados no SINASC. Os fatores de risco foram estimados por meio de análises univariadas e multivariadas, adotando-se os modelos logísticos hierarquizados. Os principais determinantes da mortalidade infantil foram os fatores biológicos (Apgar, baixo peso ao nascer, prematurida- de e presença de malformação congênita) mediados pelos fatores socioeconômicos (escolaridade, estado civil e raça/cor) e as condições da assistência (consultas de pré-natal). Embora se verifique concordância em parte dos determinantes da mortalidade infantil entre as cidades analisadas, alguns diferenciais regionais puderam ser observados expressando a condição iníqua da mortalidade infantil associada às desigualdades nas condições socioeconômicas e de acesso aos serviços de saúde.

Mortalidade Infantil; Desigualdades em Saúde; Sistemas de Informação; Fatores de Risco 


\section{Colaboradores}

L. T. S. Maia, W. V. Souza e A. C. G. Mendes participaram da concepção original, análise dos dados, redação do artigo, revisão e crítica do trabalho.

\section{Referências}

1. Hartz ZMA, Champagne F, Leal MC, Contandriopoulos AP. Mortalidade infantil "evitável” em duas cidades do Nordeste do Brasil: indicador de qualidade do sistema local de saúde. Rev Saúde Pública 1996; 30:310-8.

2. Duarte CMR. Reflexos das políticas de saúde sobre as tendências da mortalidade infantil no Brasil: revisão da literatura sobre a última década. Cad Saúde Pública 2007; 23:1511-28.

3. Departamento de Análise de Situação em Saúde, Secretaria de Vigilância em Saúde, Ministério da Saúde. Saúde Brasil 2006: uma análise da situação de saúde no Brasil. Brasília: Ministério da Saúde; 2006. (Série G. Estatística e Informação em Saúde).

4. Almeida MF, Mello-Jorge MH. O uso da técnica de "linkage" de sistemas de informação em estudos de coorte sobre mortalidade neonatal. Rev Saúde Pública 1996; 30:141-7.

\section{Agradecimentos}

Agradecemos à Secretaria de Vigilância em Saúde do Ministério da Saúde, por ter tornado os dados disponíveis e pelo financiamento da pesquisa. Ao grupo de pesquisa do Laboratório de Análise de Sistemas de Informações em Saúde, Centro de Pesquisas Aggeu Magalhães, Fundação Oswaldo Cruz (LABSIS/CPqAMFiocruz), Domício, Luiz Cláudio, Mazé e Gabriella pelas discussões sobre as informações do estudo, mas sobretudo pela convivência e aprendizado. À Maria José Guimarães, Ana Brito e Cyntia Braga pelas críticas e sugestões que tanto enriqueceram esse trabalho.
5. Camargo Jr. KR, Coeli CM. Reclink: aplicativo para o relacionamento de bases de dados, implementando o método probabilistic record linkage. Cad Saúde Pública 2000; 16:439-47.

6. Machado CJ. A literature review of record linkage procedures focusing on infant health outcomes. Cad Saúde Pública 2004; 20:362-71.

7. Camargo Jr. KR, Coeli CM. Avaliação de diferentes estratégias de blocagem no relacionamento probabilístico de registro. Rev Bras Epidemiol 2002; 5:185-96.

8. Victora CG, Huttly SR, Fuchs SC, Olinto MT. The role of conceptual frameworks in epidemiological analysis: a hierarchical approach. Int J Epidemiol 1997; 26:224-7.

9. Castro MSM, Vieira VA, Assunção RM. Padrões espaço-temporais da mortalidade por câncer de pulmão no Sul do Brasil. Rev Bras Epidemiol 2004; 7:131-43. 
10. Barros FC, Victora CG. Maternal-child health in Pelotas, Rio Grande do Sul State, Brazil: major conclusions from comparisons of the 1982, 1993, and 2004 birth cohorts. Cad Saúde Pública 2008; 24 Suppl 3:S461-7.

11. Morais Neto OL, Barros MBA. Fatores de risco para mortalidade neonatal e pós-neonatal na Região Centro-Oeste do Brasil: linkage entre bancos de dados de nascidos vivos e óbitos infantis. Cad Saúde Pública 2000; 16:477-85.

12. Jobim R, Aerts D. Mortalidade infantil evitável e fatores associados em Porto Alegre, Rio Grande do Sul, Brasil, 2000-2003. Cad Saúde Pública 2008; 24:179-87.

13. Silva CF, Leite AJM, Almeida NMGS, Gondim RC. Fatores de risco para mortalidade infantil em município do Nordeste do Brasil: linkage entre bancos de dados de nascidos vivos e óbitos infantis - 2000 a 2002. Rev Bras Epidemiol 2006; 9:69-80.

14. França E, Souza JM, Guimarães MDC, Goulart EMA, Colosimo E, Antunes CMF. Associação entre fatores sócio-econômicos e mortalidade infantil por diarréia, pneumonia e desnutrição em região metropolitana do Sudeste do Brasil: um estudo caso-controle. Cad Saúde Pública 2001; 17:1437-47.

15. Matijasevich A, Victora CG, Barros AJD, Santos IS, Marco PL, Albernaz EP, et al. Widening ethnic disparities in infant mortality in Southern Brazil: comparison of 3 birth cohorts. Am J Public Health 2008; 98:692-8

16. Menezes AMB, Hallal PC, Santos IS, Victora CG, Barros FC. Infant mortality in Pelotas, Brazil: a comparison of risk factors in two birth cohorts. Rev Panam Salud Pública 2005; 18:439-46.

17. Hauck FR, Tanabe KO, Moon RY. Racial and ethnic disparities in infant mortality. Semin Perinatol 2010; 35:209-20.

18. Departamento de Análise de Situação de Saúde, Secretaria de Vigilância em Saúde, Ministério da Saúde. Vigilância em saúde: dados e indicadores selecionados. Brasília: Ministério da Saúde; 2004.

19. Araújo BF, Bozzetti MC, Tanaka ACA. Mortalidade neonatal no município de Caxias do Sul: um estudo de coorte. J Pediatr (Rio J.) 2000; 76:200-6.

20. Victora CG. Intervenções para reduzir a mortalidade infantil pré-escolar e materna no Brasil. Rev Bras Epidemiol 2001; 4:3-69.
21. Nascimento EMR, Costa MCN, Mota ELA, Paim JS. Estudo de fatores de risco para óbitos de menores de um ano mediante compartilhamento de bancos de dados. Cad Saúde Pública 2008; 24: 2593-602.

22. Martins EF, Velásquez-Meléndez G. Determinantes da mortalidade neonatal a partir de uma coorte de nascidos vivos, Montes Claros, Minas Gerais, 1997-1999. Rev Bras Saúde Matern Infant 2004; 4: 405-12.

23. Kilsztajn S, Lopes ES, Carmo MSN, Reyes AMA. Vitalidade do recém-nascido por tipo de parto no Estado de São Paulo, Brasil. Cad Saúde Pública 2007; 23:1886-92.

24. Ribeiro VS, Silva AAM, Barbieri MA, Bettiol H, Aragão VMF, Coimbra LC, et al. Infant mortality: comparison between two birth cohorts from Southeast and Northeast, Brazil. Rev Saúde Pública 2004; 38:773-9.

25. Caldeira AP, França E, Goulart EMA. Mortalidade infantil pós-neonatal e qualidade da assistência médica: um estudo caso-controle. J Pediatr (Rio J.) 2001; 77:461-8.

26. Aquino TA, Guimarães MJB, Sarinho SW, Ferreira LOC. Fatores de risco para a mortalidade perinatal no Recife, Pernambuco, Brasil, 2003. Cad Saúde Pública 2007; 23:2853-61.

27. Machado CJ, Hill K. Maternal, neonatal and community factors influencing neonatal mortality in Brazil. J Biosoc Sci 2005; 37:193-208.

28. d'Orsi E, Carvalho MS, Cruz OG. Similarity be tween neonatal profile and socioeconomic index: a spatial approach. Cad Saúde Pública 2005; 21:786-94.

29. Shimakura SE, Carvalho MS, Aerts DRGC, Flores R. Distribuição espacial do risco: modelagem da mortalidade infantil em Porto Alegre, Rio Grande do Sul, Brasil. Cad Saúde Pública 2001; 17:1251-61.

30. Ribeiro AM, Guimarães MJ, Lima MC, Sarinho SW, Coutinho SB. Fatores de risco para mortalidade neonatal em crianças com baixo peso ao nascer. Rev Saúde Pública 2009; 43:246-55.

31. Wise PH. Efficacy and justice: the importance of medical research and tertiary care to social disparities in infant mortality. J Perinatol 1999; 19(6 Pt 2):S24-7.

Recebido em 04/Out/2011

Versão final reapresentada em 23/Mai/2012

Aprovado em 30/Jul/2012 